\title{
Wideband microstrip patch antenna loaded with parasitic metal strips and coupling slots
}

\author{
Yufan Cao ${ }^{1 a)}$, Yang Cai ${ }^{2}$, Wenquan Cao ${ }^{1}$, Zuping Qian ${ }^{1}$, \\ and Lei $\mathbf{Z h u}^{1}$ \\ ${ }^{1}$ College of Communications Engineering, Army Engineering University of PLA, \\ Nanjing 210007, China \\ 2 Space Engineering University, Beijing 101400, China \\ a)caoyufan135@sina.com
}

\begin{abstract}
A novel microstrip patch antenna loaded with parasitic metal strips is proposed. Two identical parasitic metal strips are asymmetrically located along with the two radiating edges of a conventional patch antenna. Two coupling slots are etched adjacent to the strips on the ground plane to realize energy transmission. Therefore, a new resonant mode $\mathrm{TM}_{30}$ mode generated by parasitic metal strips together with the original $\mathrm{TM}_{10}$ mode result in wide impedance bandwidth. Experimental results indicate that an impedance bandwidth of $23 \%$ from 13.8 to $17.4 \mathrm{GHz}$ is obtained, with a low profile less than $\lambda_{0} / 20$. Moreover, the proposed antenna presents $1-\mathrm{dB}$ gain flatness across the operating bandwidth.
\end{abstract}

Keywords: microstrip patch antenna, parasitic strips, wideband

Classification: Microwave and millimeter-wave devices, circuits, and modules

\section{References}

[1] K.-F. Lee and K.-F. Tong: "Microstrip patch antennas basic characteristics and some recent advances," Proc. IEEE 100 (2012) 2169 (DOI: 10.1109/JPROC. 2012.2183829).

[2] D. H. Schaubert, et al: "Effect of microstrip antenna substrate thickness and permittivity: comparison of theories and experiment," IEEE Trans. Antennas Propag. 37 (1989) 677 (DOI: 10.1109/8.29353).

[3] Y. Kazama, et al:: "Vertical polarization single-feed dual-frequency microstrip antenna with an arc-shaped slot," IEICE Electron. Express 2 (2005) 60 (DOI: 10.1587/elex.2.60).

[4] K. M. Luk, et al.: "Broadband microstrip patch antenna," Electron. Lett. 34 (1998) 1442 (DOI: 10.1049/el:19981009).

[5] V. P. Sarin, et al.: "A wideband stacked offset microstrip antenna with improved gain and low cross polarization," IEEE Trans. Antennas Propag. 59 (2011) 1376 (DOI: 10.1109/TAP.2011.2109362).

[6] J. A. Ansari, et al:: "Analysis of wideband multilayer patch antenna with two parasitic elements," Microw. Opt. Technol. Lett. 51 (2009) 1397 (DOI: 10. 1002/mop.24378). 
[7] Z. Wu, et al.: "Dual-band antenna integrating with rectangular mushroom-like superstrate for WLAN applications," IEEE Antennas Wireless Propag. Lett. 15 (2016) 1269 (DOI: 10.1109/LAWP.2015.2504558).

[8] H. Kang and S. O. Park: "Mushroom meta-material based substrate integrated waveguide cavity backed slot antenna with broadband and reduced back radiation," IET Microw. Antennas Propag. 10 (2016) 1598 (DOI: 10.1049/ietmap.2016.0056).

[9] S. H. Wi, et al.: "Wideband microstrip patch antenna with U-shaped parasitic elements," IEEE Trans. Antennas Propag. 55 (2007) 1196 (DOI: 10.1109/TAP. 2007.893427).

[10] T. H. Jang, et al:: "A wideband aperture efficient 60-GHz series-fed E-shaped patch antenna array with copolarized patches," IEEE Trans. Antennas Propag. 64 (2016) 5518 (DOI: 10.1109/TAP.2016.2621023).

\section{Introduction}

Microstrip patch antenna has attracted serious attention since the late 1970s, which offers the acknowledged advantages of low profile, ease of fabrication, easy integration with other planar circuits, and etc. It is known that the microstrip patch antenna works in a quite narrow bandwidth, typically less than $5 \%$, which limits its practical applications [1]. Series of methods have been proposed to improve the bandwidth of microstrip patch antenna. In general, two basic methods are employed to broaden the bandwidth. One is to use thick substrate with low permittivity $[2,3,4]$ and the other is to stack patches on the different layers $[5,6]$. Besides, due to the unusual electromagnetic properties of metamaterial, kinds of metamaterial-based antennas have been proposed to achieve wideband performance [7, 8].

Since the parasitic elements possess the advantage of exciting new resonant modes, a great number of wideband antennas are achieved by employing parasitic elements methods. Through incorporating two U-shaped parasitic elements into the radiating edges of a patch, an impedance bandwidth of $27.3 \%$ was obtained [9]. A novel series-fed patch antenna array with copolarized parasitic patches was designed in [10], and the operating bandwidth was from 57 to $66 \mathrm{GHz}$.

In this paper, a new wideband patch antenna adopting parasitic elements is studied. Through loading two identical parasitic metal strips along with the two radiating edges of a conventional patch antenna, two resonant modes are simultaneously excited, resulting in broad bandwidth. What's more, two coupling slots are etched on the ground plane realize energy transmission.

\section{Antenna design}

\subsection{Antenna geometry}

The geometry of the proposed microstrip patch antenna is shown in Fig. 1, which consists of three parts: a conventional patch with probe feeding, two parasitic metal strips asymmetrically placed along with the two radiating edges of the patch, and two coupling slots etched adjacent to the strips on the ground plane. The conventional patch occupies an area of $l \times w$ and the feeding probe is located with an 
offset distance of $d_{f}$ from the center of the patch. The two parasitic metal strips with same width $s$ are placed next to the patch in y-direction with gaps $d_{0}$ and $d_{1}$, respectively. Two identical slots with width $g$ are etched on the ground plane, which are adjacent to the strips. This antenna is constructed on a 1.016-mm-thickness RT/Rogers 5880 substrate with permittivity of 2.2 and loss tangent of 0.0009 .

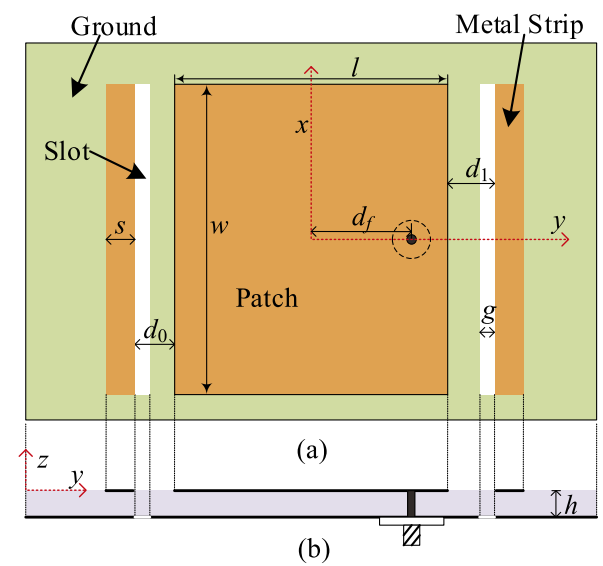

Fig. 1. Geometrical configuration of the proposed patch antenna. (a) Top view. (b) Side view.

\subsection{Mode analysis}

The simulated $\left|S_{11}\right|$ of the proposed antenna and the conventional patch antenna are compared in Fig. 2. It is illustrated that the impedance bandwidth of conventional patch antenna is very narrow. After loading two parasitic metal strips along with the radiating edges of the patch antenna, a new resonant frequency is excited, which is close to the original resonant frequency so that the bandwidth is significantly broadened.

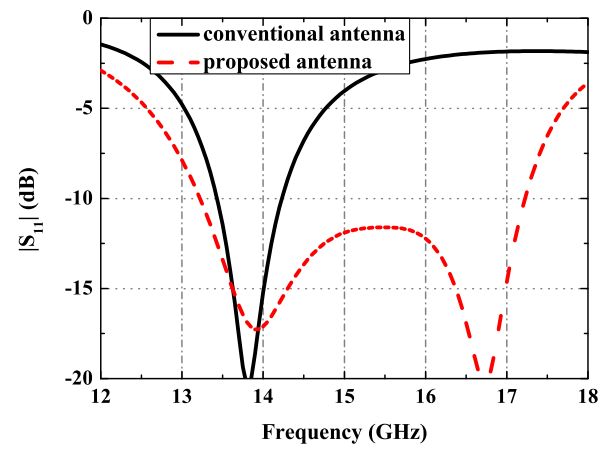

Fig. 2. Simulated $\left|S_{11}\right|$ of the proposed antenna and the conventional patch antenna.

In order to demonstrate the working mechanism of the proposed antenna in theory, the electric field distributions at resonant frequencies are researched.

The simulated electric field distribution at resonant frequency $13.95 \mathrm{GHz}$ is depicted in Fig. 3. It is observed that the electric field distribution proves the $\mathrm{TM}_{10}$ mode generated by the conventional patch antenna. Few energy is coupled to the 
parasitic metal strips, which can be neglected. In other words, the loaded parasitic strips take no effect on radiation at lower frequencies.

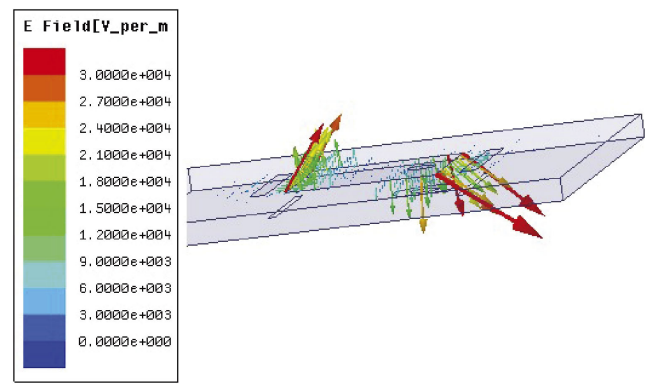

(a)

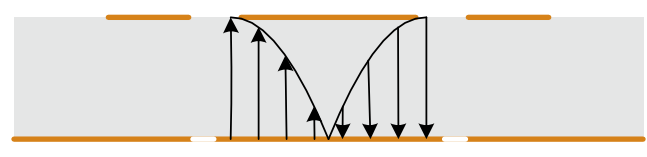

(b)

Fig. 3. $\mathrm{TM}_{10}$ mode at resonant frequency $13.95 \mathrm{GHz}$. (a) Simulated electric field distribution. (b) Sketch of the operation mechanism.

Fig. 4 shows the simulated electric field distribution at resonant frequency 16.75 GHz. The simulated electric field distribution is similar to the $\mathrm{TM}_{30}$ mode. The energy is radiated to free space not only by the conventional patch but also by the parasitic strips. Meanwhile, the coupling slots is crucial for the energy transmission from patch to metal strips. Finally, the $\mathrm{TM}_{30}$ mode generated at higher frequencies together with the $\mathrm{TM}_{10}$ mode generated at lower frequencies result in wide bandwidth.

\subsection{Location of metal strips}

In our original design, the loaded parasitic metal strips are symmetrically arranged on both sides of the patch. The two gaps are with same size, i.e., $d_{0}=d_{1}$. Simulation results show that wide bandwidth is obtained. However, the far field radiation performances are defective. Simulated 3-D radiation pattern of the patch antenna loaded with symmetrically placed metal strips at $15 \mathrm{GHz}$ is depicted in Fig. 5. It is obvious that the maximum radiation direction deviates from z-direction but moves to y-direction slightly. Since the location of the feeding probe is not in the center of the patch, the two parasitic metal strips are asymmetrical about the feeding point. Therefore, the maximum radiation direction migrates from z-direction to y-direction slightly due to the y-direction shifted feeding point.

In order to adjust the maximum radiation direction, the parasitic metal strip in y-direction should be moved far away from the patch, i.e., $d_{0}<d_{1}$. The simulated gains of the patch antenna loaded with symmetrically and asymmetrically placed metal strips at z-direction are compared in Fig. 6. It is observed that the average gain of the proposed antenna is improved more than $1 \mathrm{~dB}$ compared to that with symmetrically placed metal strips. 


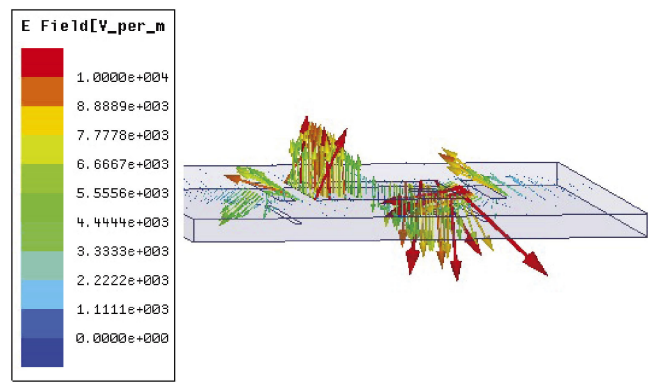

(a)

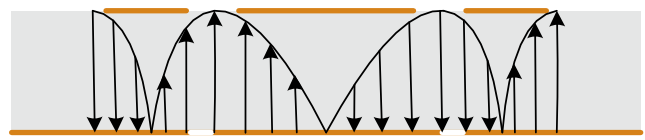

(b)

Fig. 4. $\mathrm{TM}_{30}$ mode at resonant frequency $16.75 \mathrm{GHz}$. (a) Simulated electric field distribution. (b) Sketch of the operation mechanism.

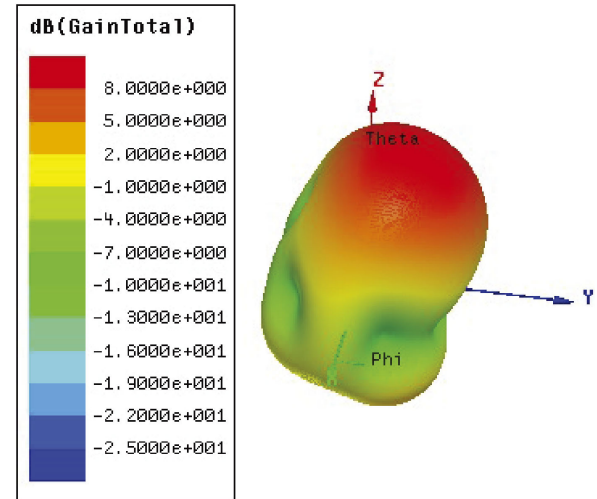

Fig. 5. Simulated 3-D radiation pattern of the patch antenna loaded with symmetrically placed metal strips at $15 \mathrm{GHz}$.

\subsection{Parameters analysis}

The constitutive parameters of the parasitic metal strips and coupling slots are studied, as shown in Fig. 7. The effect of different gap lengths $d_{0}$ on the resonant frequencies are illustrated in Fig. 7(a). With the increase of the $d_{0}$, the increased lower resonant frequency together with the decreased higher resonant frequency make a narrow operating bandwidth.

Fig. 7(b) depicts the simulated $\left|S_{11}\right|$ versus the other gap lengths $d_{1}$. With the increase of the $d_{1}$, the lower resonant frequency keeps constant and the higher resonant frequency decreases slightly, resulting in a relatively narrow operating bandwidth. Through optimizing the size of two gaps $d_{0}$ and $d_{1}$, a desired working bandwidth is obtained.

The coupling slots have an important effect on the impedance matching performance, as shown in Fig. 7(c). With the increase of the slots width $g$, the impedance matching is improved but the operating bandwidth is decreased. 


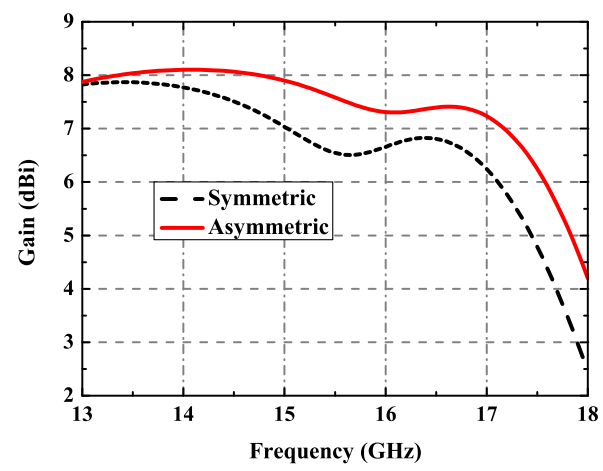

Fig. 6. Simulated gains of the patch antenna loaded with symmetrically and asymmetrically placed metal strips at z-direction.

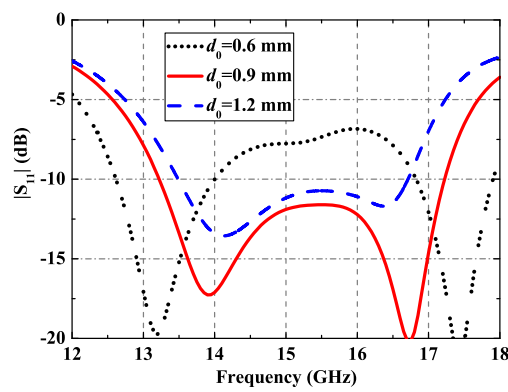

(a)

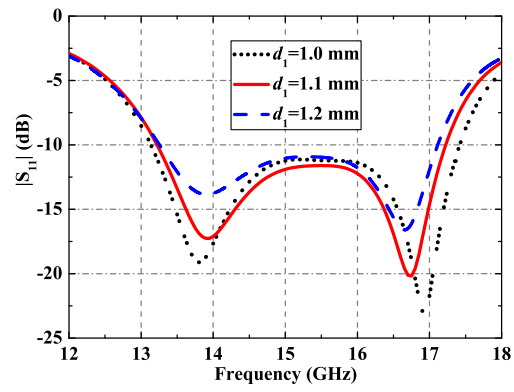

(b)

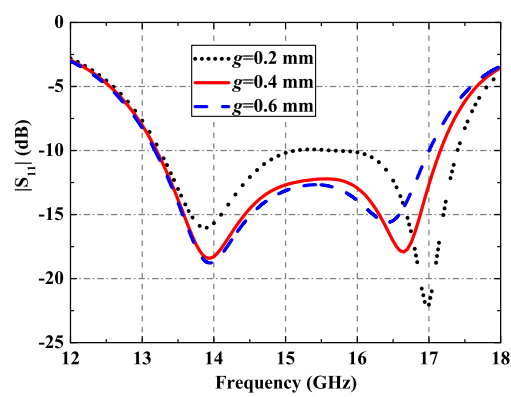

(c)

Fig. 7. Simulated $\left|S_{11}\right|$ of the proposed antenna with different parameters. (a) gap $d_{0}$. (b) gap $d_{1}$. (c) slot width $g$.

\section{Experimental results}

The proposed patch antenna is optimized by using the HFSS software and fabricated for verification. Fig. 8 depicts the photograph of the proposed antenna. The optimized dimensions of the prototype are: $l=6.6 \mathrm{~mm}, w=6.8 \mathrm{~mm}, d_{f}=$ $2.1 \mathrm{~mm}, d_{0}=0.9 \mathrm{~mm}, d_{1}=1.1 \mathrm{~mm}, g=0.3 \mathrm{~mm}, s=0.7 \mathrm{~mm}, h=1 \mathrm{~mm}$. Comparison of simulated and measured reflection coefficients is given in Fig. 9. The measured impedance bandwidth is about $23 \%$ ranging from 13.8 to $17.4 \mathrm{GHz}$. Moreover, the measured results show good accordance with simulated ones. The slight frequency shift is attributed to the fabrication tolerance.

Fig. 10 depicts the simulated and measured gains of the proposed antenna. It is obvious that the measured results agree with the simulated ones. What's more, 1-dB gain flatness is obtained over the operating bandwidth. Since the coupling slots are 


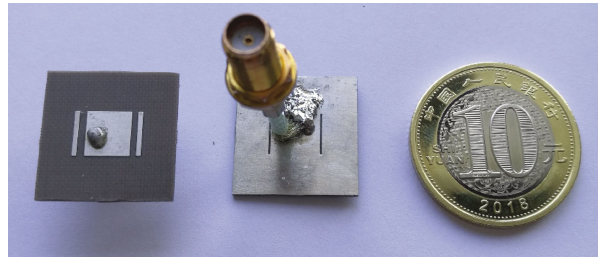

Fig. 8. Photograph of the proposed patch antenna.

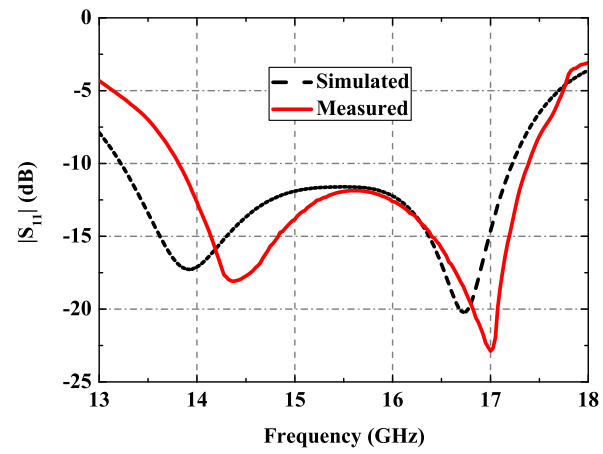

Fig. 9. Simulated and measured $\left|S_{11}\right|$ of the proposed antenna.

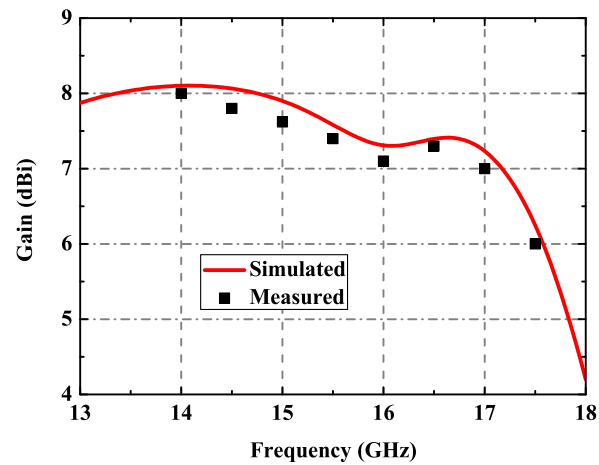

Fig. 10. Simulated and measured gains of the proposed antenna.

etched on the ground plane, the gains decrease at higher frequencies due to the enhanced backward radiation.

The simulated and measured far field radiation patterns at three representative frequencies across the entire bandwidth are illustrated in Fig. 11. Good agreements are observed between the simulated results and the measured ones. Stable broadside radiation patterns for the patch antenna are obtained. However, with the increase of frequencies, the backward radiation increases, which explains the decreased gains in Fig. 10. When energy is transmitted from patch to metal strips at high frequencies, a part of energy leaks from the coupling slots and backward radiation is improved. All in all, the main radiation direction maintains broadside direction. 


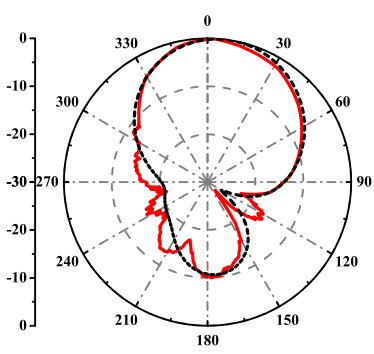

(a)

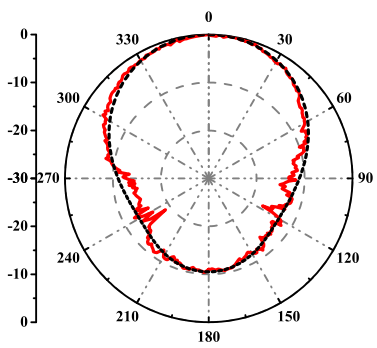

(d)

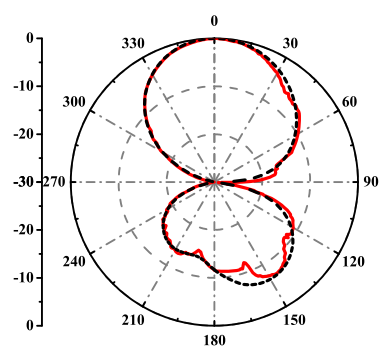

(b)

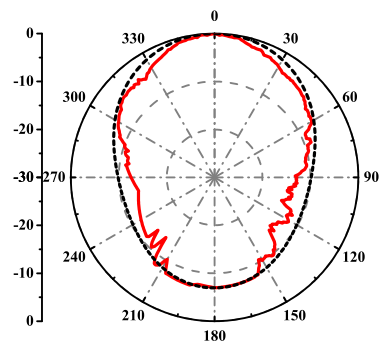

(e)

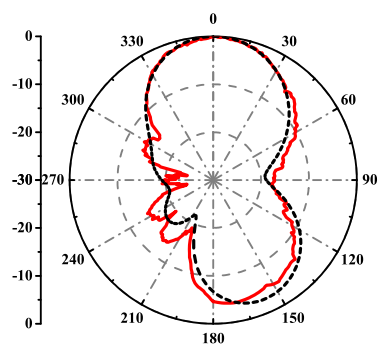

(c)

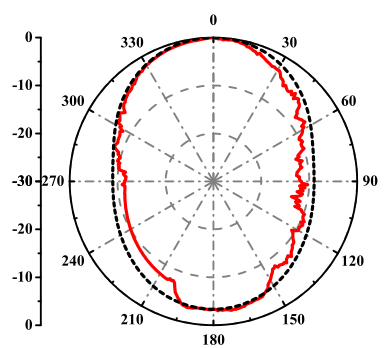

(f)

Fig. 11. Simulated and measured far field radiation patterns of the proposed antenna at three representative frequencies. (a) Eplane at $14 \mathrm{GHz}$. (b) E-plane at $15.5 \mathrm{GHz}$. (c) E-plane at $17 \mathrm{GHz}$. (d) H-plane at $14 \mathrm{GHz}$. (e) H-plane at $15.5 \mathrm{GHz}$. (f) H-plane at $17 \mathrm{GHz}$. (Dashed black lines denote the simulated results and straight red lines denote the measured results.)

\section{Conclusion}

In this paper, a novel wideband microstrip patch antenna is studied. By loading two parasitic metal strips along with two radiating edges of the patch, a new generated $\mathrm{TM}_{30}$ mode and the original $\mathrm{TM}_{10}$ mode lead to wide impedance bandwidth. Measured results show that the improved bandwidth is about $23 \%$ and a good gain flatness is achieved. Besides, the proposed antenna maintains stable broadside radiation patterns over the entire operating bandwidth. Above all, the proposed antenna offers good advantages of wide bandwidth, compact structure, low profile and ease of fabrication.

\section{Acknowledgments}

This work has been supported by the National Science Foundation of China under Grant 61271103. 